\title{
Characterisation of the effector cells responsible for the in vitro cytotoxicity of blood leucocytes from aphthous ulcer patients for oral epithelial cells
}

\author{
D W Thomas, J Bagg, D M Walker
}

\begin{abstract}
This study was designed to identify the cells responsible for the spontaneous cell mediated cytotoxic effect (SCMC) exerted by peripheral blood leucocytes from patients with recurrent aphthous ulceration, towards cultured oral epithelial cells. Peripheral blood leucocytes from recurrent aphthous ulceration patients exerted a significantly greater $(\mathbf{p}<0.01)$ degree of cytotoxicity towards the oral epithelial target cells than did peripheral blood leucocytes from healthy control subjects, or from patients with non-specific ulceration. Depletion of CD-5 positive cells (T-lymphocytes) resulted in a significant decrease in the SCMC in aphthous patients. Depletion of CD-16 positive cells (NK-cells) produced no significant change in cytotoxicity. $T$-lymphocytes, therefore, appear to be intimately involved in the in vitro SCMC effect in recurrent aphthous ulceration.
\end{abstract}

Recurrent aphthous ulceration' is a form of focal oral ulceration, which affects up to $20 \%$ of the population. Although recurrent aphthous ulceration may rarely be a marker of underlying systemic illness such as coeliac disease, or present as a feature of Behçet's syndrome, in most cases no additional body systems are affected, and patients remain otherwise fit and well.

An immunopathogenesis for recurrent aphthous ulceration was first suggested by Lehner, ${ }^{2}$ who likened the histological appearance of aphthous ulceration, with its early mononuclear cell infiltrate, to that of a type IV hypersensitivity reaction. The finding of significant lymphocytotoxicity in other immunologically mediated diseases $^{3}$ prompted Dolby ${ }^{4}$ to show that peripheral blood leucocytes from aphthous patients exerted a significantly enhanced cytotoxicity in vitro, for allogeneic gingival epithelial cells. This finding has been substantiated by other workers. ${ }^{5}$ Greenspan et $a l^{7}$ and Burnett and Wray, ${ }^{8}$ however, found that the increased cytotoxicity was antibody dependent, presumably mediated by $\mathrm{K}$ or NK cells.

Suppressor/cytotoxic $\mathrm{T}$ cells, $\mathrm{T}$ helper cells and NK cells have been identified in the infiltrate in aphthae, in different proportions varying with the duration of the lesion. ${ }^{9}$ Despite much speculation, however, the effector cells mediating the tissue damage seen in recurrent aphthous ulceration remain unidentified.

The aims of the present study were three-fold. Firstly, the cytotoxic effect of peripheral blood leucocytes from recurrent aphthous ulceration sufferers on epithelial target cells derived from a novel source, namely, actively growing longterm cultures of oral epithelium, was investigated. Secondly, the specificity of the raised SCMC in recurrent aphthous ulceration was examined by determining the cytotoxic effect of peripheral blood leucocytes from patients with other types of mouth ulcers. This part of the study was designed to show whether or not the previously described cytotoxic process in recurrent aphthous ulceration was merely an epiphenomenon related to oral epithelial cell breakdown. Finally, tentative identification of the cytotoxic effector cell in our in vitro system was attempted by studying the effect on cytotoxicity of leucocyte subset depletion.

\section{Methods}

PATIENTS WITH RECURRENT APHTHOUS ULCERATION

Thirty two patients were studied, each suffering from minor aphthous ulceration, diagnosed according to the criteria of Lehner, ${ }^{10}$ of recurrent crops of one to five small painful ulcers affecting the non-keratinised oral mucosa and healing within four to 14 days, without scarring or a systemic mucocutaneous disorder. Seventeen of the patients were men and 15 were women, aged from 16 to 56 years (mean 30.7). Seventeen of the patients had ulcers at the time of study whilst the remainder were ulcer free (remission), though all patients were experiencing regular bouts of ulceration. All of the patients were haematologically normal and only two were tobacco smokers.

\section{DISEASED CONTROLS}

We also investigated as diseased controls six patients with other types of active mouth ulceration, due to trauma (four patients), erythema multiforme (one) and erosive lichen planus (one) with ages ranging from 27 to 63 years (mean age $44 \cdot 6$ years).

\section{HEALTHY CONTROL SUBJECTS}

For each aphthous or diseased control patient, an age and sex matched healthy volunteer was studied in the same experiment.

COLLECTION OF BLOOD AND SEPARATION OF PERIPHERAL BLOOD MONONUCLEAR CELLS

Twenty millilitres of venous blood were collected from the antecubital fossa of aphthous and 
control patients into heparinised tubes (Vacutainer Systems UK, Becton Dickinson). The peripheral blood mononuclear cells were separated by density gradient centrifugation over Ficoll Hypaque." The leucocytes were washed twice in phosphate buffered saline (PBS) and their concentration adjusted to $3 \times 10 \% \mathrm{ml}$ in growth medium, before use in the cytotoxicity assay.

CULTURE OF ORAL EPITHELIAL CELLS Oral mucosa was harvested from patients undergoing routine minor oral surgical procedures. Within one to four hours after collection, the tissue was transported to the laboratory in Dulbecco's modified Eagle's medium $(10 \times)$ containing fetal calf serum $(10 \%)$, penicillin/streptomycin mixture, $1000 \mathrm{IU} / \mathrm{ml}$ and amphotericin, $5000 \mathrm{IU} / \mathrm{ml}$.

Most of the underlying connective tissue was removed and the epithelium and remaining connective tissue were minced finely under aseptic conditions using Iris scissors in a laminar air flow cabinet. These diced explants were resuspended in a growth medium identical with transport medium, but with added hydrocortisone $(1 \mathrm{mg} / \mathrm{ml})$ and cholera toxin $(0.002 \mathrm{mg} / \mathrm{ml})$. The explants were seeded out in tissue culture flasks containing a monolayer of 3T3 fibroblasts, which acted as a feeder layer. ${ }^{12}$ Division of the 3T3 cells was halted by treatment with mitomycin C 12-24 hours before culturing the epithelial explants. The cultures were maintained at $37^{\circ} \mathrm{C}$ in a $5 \% \mathrm{CO}_{2}$ atmosphere, removing any non-adherent explants and changing the medium after 72 hours and thereafter twice weekly.

CHARACTERISATION OF TARGET CELLS The cultured cells were characterised as epithelial cells by three independent methods:

\section{Scanning electron microscopy}

Cultured cells were fixed in $3 \%$ glutaraldehyde at $4^{\circ} \mathrm{C}$ in Sorenson's buffer, dehydrated through graded alcohols, and critical point dried from Arklone (ICI plc, Runcorn, Cheshire, UK). They were mounted on stubs and sputter-coated with gold (thickness $48 \mathrm{~nm}$ ), before examination in an ISI Super III scanning electron microscope.

\section{Indirect immunofluorescence}

Twenty microlitres of pemphigus serum diluted $1: 50$ in PBS were added to cultured oral epithelial cells suspended in a minimal volume of PBS. After 45-60 minutes incubation at ambient temperature, the cells were washed by centrifuging at $3000 \mathrm{rpm}$ in PBS and reincubated for a further 45 minutes with FITC-labelled antihuman immunoglobulin (Dakopatts Limited, High Wycombe, Buckinghamshire, UK).

\section{Immunoperoxidase staining}

Smear preparations of the epithelial cultures, with fibroblasts as negative controls, were fixed in acetone for five minutes at $4^{\circ} \mathrm{C}$. All subsequent incubations were carried out at ambient temperature and separated by three washes in PBS. The preparations were treated with $3 \%$ hydrogen peroxide in methanol for five minutes to block endogenous peroxidase, followed by incubation with normal swine serum for 20 minutes. The slides were then treated sequentially with the primary rabbit antihuman keratin antibody ( 20 min), sheep antirabbit immunoglobulin (20 min) and peroxidase-rabbit antiperoxidase $(20 \mathrm{~min})$ (all from Orthodiagnostic Systems Inc, Raritan, New Jersey). Finally, the slides were incubated for 40 minutes with diaminobenzidine ${ }^{13}$ to visualise any labelled antigens.

\section{CYTOTOXICITY ASSAY}

The primary cultures of oral epithelial cells were harvested by dispersion with a mixture of trypsin $(0.05 \%)$ and EDTA $(0.02 \%)$ for four to five minutes, or until the cells lost their adherence to the flask. Trypsinisation was halted by the addition of Dulbecco's modified Eagle's medium containing $20 \%$ fetal calf serum. The cells were then washed twice in medium and resuspended in growth medium at a concentration of $7.5 \times 10^{4}$ cells $/ \mathrm{ml}$. Only preparations in which at least $80 \%$ of the epithelial cells were viable were used, and the same preparation of target cells was used for both control and experimental groups in any one run. One hundred microlitres of the leucocyte suspension were added to $20 \mu$ lof the epithelial cells, thus providing a leucocyte:epithelial cell ratio of 200:1. At this ratio, the largest difference in cytotoxicity between aphthous and control leucocytes could be demonstrated (unpublished data). As a control, $100 \mu \mathrm{l}$ of growth medium alone was added to $20 \mu \mathrm{l}$ of the epithelial cell suspension. Triplicate cultures were incubated at $37^{\circ} \mathrm{C}$ in a humidified incubator with $5 \% \mathrm{CO}_{2}$ in air for 17 hours. The cells were sedimented by centrifugation, $80 \mu \mathrm{l}$ of the supernatant removed, and $40 \mu \mathrm{l}$ of ethidium bromide and acridine orange added to the remaining $40 \mu$ l of cell suspension. The viability of 200 of the epithelial cells per culture was assessed blind by a single examiner in coded cultures by direct ultraviolet microscopy.

\section{LEUCOCYTE SUBSET DEPLETION}

Leucocyte subset depletion was effected by complement dependent lysis of the peripheral blood leucocyte preparations with the monoclonal antibodies anti Leu-1 and anti Leu-11 (Becton Dickinson Immunocytochemistry Systems, Mountain View, California, 94039). Anti Leu-1 is specific for peripheral blood leucocytes expressing the CD 5 antigen (T-lymphocytes) while anti Leu-11 recognises the CD16 antigen (natural killer $(\mathrm{NK})$ cells, and killer $(\mathrm{K})$ cells).

Fifty microlitre aliquots of the leucocyte suspension were incubated with $500 \mu$ l of a 1:100 dilution of the appropriate monoclonal antibody for 50 minutes on ice, and recovered by centrifugation. Preliminary experiments showed that complement at a dilution of $1: 10$ gave optimal cell lysis. The leucocytes were then incubated, therefore, with $100 \mu \mathrm{l}$ of a $1: 10$ dilution of baby 
rabbit complement at $37^{\circ} \mathrm{C}$ for $50 \mathrm{~min}$, pelleted by centrifugation and washed twice in phosphate buffered saline. The treated leucocytes were spun through $10 \%$ fetal calf serum, counted and the concentration of viable cells adjusted in growth medium to the original concentration of $3 \times 10^{6}$ cells $/ \mathrm{ml}$. The viability of all leucocyte preparations after depletion was greater than $80 \%$. The peripheral blood leucocytes were then incubated with the oral epithelial target cells in the above cytotoxicity assay.

\section{CYTOTOXICITY INDICES AND STATISTICAL} ANALYSIS

The data were presented as the mean number of surviving cultured oral epithelial cells. In addition, a modified version of the 'difference in cytotoxicity index' $(\bigwedge \mathrm{CT})^{5}$ was determined:

Number of epithelial Number of epithelial cells surviving in cells surviving in presence of control - presence of aphthous leucocytes leucocytes

Number of epithelial cells surviving in presence of medium alone

Thus, positive values for $\wedge \mathrm{CT}$ indicate that aphthous leucocytes are more cytotoxic than are the control leucocytes. A paired analysis of variance, the $F$ test, was used to analyse the data obtained. This allowed an analysis of intergroup and intertest variation.

\section{Results}

CHARACTERISATION OF TARGET CELLS

Scanning electron microscopy confirmed that the cultured oral mucosal explants had the ultrastructural features of epithelial cells. The cells formed a well defined monolayer with prominent nuclei. The cells also reacted positively with the rabbit antihuman keratin using the immunoperoxidase method and demontrated well defined positive surface immunofluorescence after incubation with the human pemphigus serum. The control fibroblasts reacted negatively in both these tests.

CYTOXICITY OF BLOOD LEUCOCYTES FROM APHTHOUS PATIENTS AND CONTROL

SUBJECTS

The leucocytes from 32 patients with aphthous ulceration were significantly more cytotoxic for oral epithelial cells when compared with leucocytes from healthy control individuals $(\mathrm{p}<0.001)$ (Fig 1). By paired analysis of variance, the difference in cytotoxicity between five aphthous patients and their matched controls was found to be significantly greater than that within each triplicate $(\mathrm{p}<0.05)$.

LEUCOCYTE CYTOTOXICITY AND ACTIVITY OF APHTHOUS ULCERATION

The leucocyte cytotoxicity $(\bigwedge \mathrm{CT}$ ) values were positive in $31 / 32$ patients and were significantly higher $(p<0.01)$ for aphthous patients with

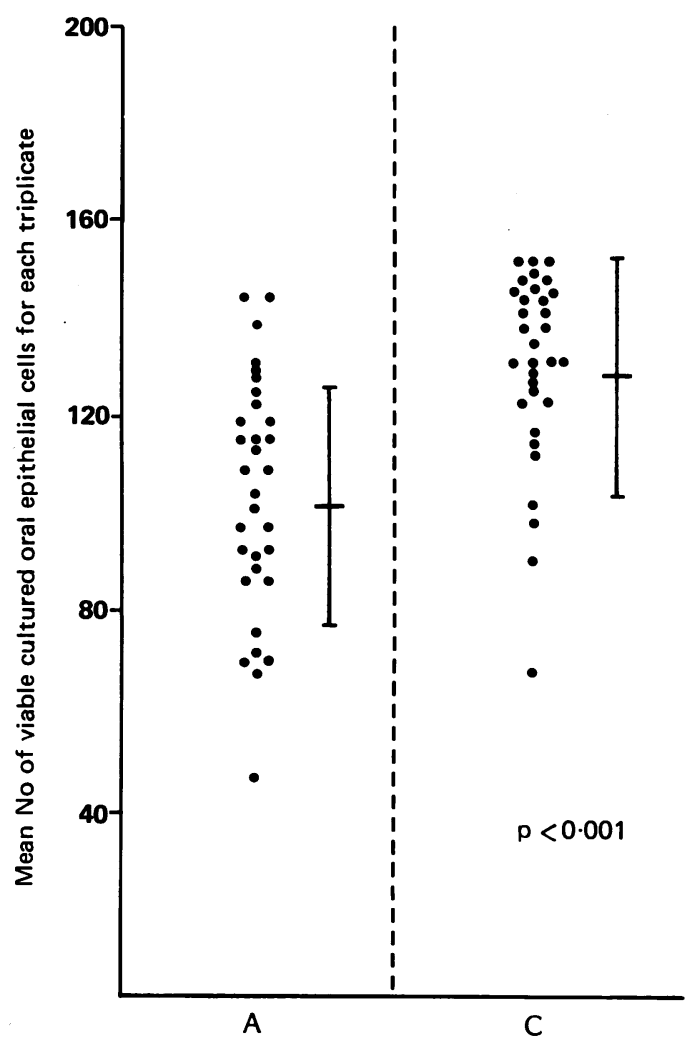

Figure 1: Mean number of cultured viable oral epithelial cells after incubation with leucocytes from aphthous patients (A) and control subjects (C) at an effector:target cell ratio of 200:1. The horizontal bars represent the overall mean values and corresponding standard deviations.

mouth ulcers at the time of sampling compared with those of patients in remission (Fig 2).

\section{LEUCOCYTE CYTOTOXICITY IN PATIENTS} WITH NON-APHTHOUS MOUTH ULCERS In the six patients with other types of oral ulceration there were no statistically significant

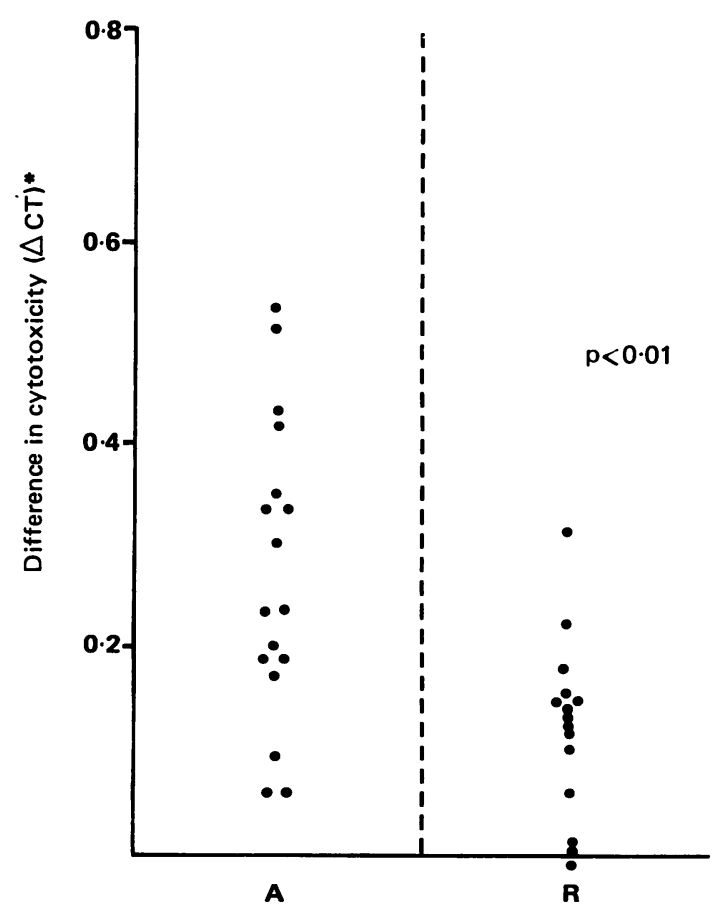

Figure 2: Mean difference in cytotoxicity values ( $\ C T)$ for leucocytes from aphthous patients with active ulceration $(\mathrm{A})$ and aphthous patients in remission $(\mathrm{R})$ towards cultured oral epithelial cells. 


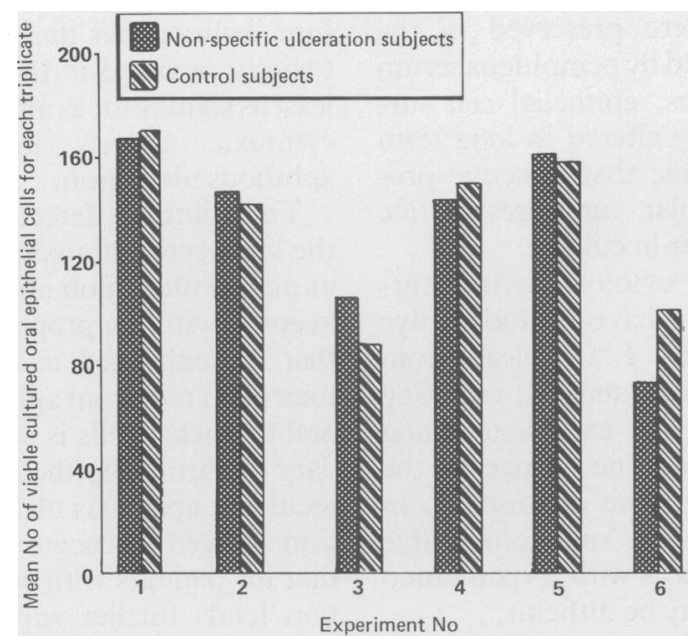

Figure 3: Mean number of cultured viable oral epithelial cells after incubation with leucocytes from patients with non-specific ulceration and control subjects.

differences between their leucocyte cytotoxicity for oral epithelial cells and that mediated by leucocytes from matched healthy control subjects (Fig 3).

\section{EFFECT OF T CELL DEPLETION ON}

CYTOTOXICITY

Leucocytes from a group of 10 patients with aphthous ulceration were significantly more cytotoxic for oral epithelial cells than were leucocytes from healthy controls $(\mathrm{p}<0 \cdot 01)$. After leucocyte treatment with the anti Leu-1 monoclonal antibody and complement, to deplete the $T$ cell population, the cytotoxic activities of both these populations were not statistically different $(\mathrm{p}>0 \cdot 1)$ (Fig 4).

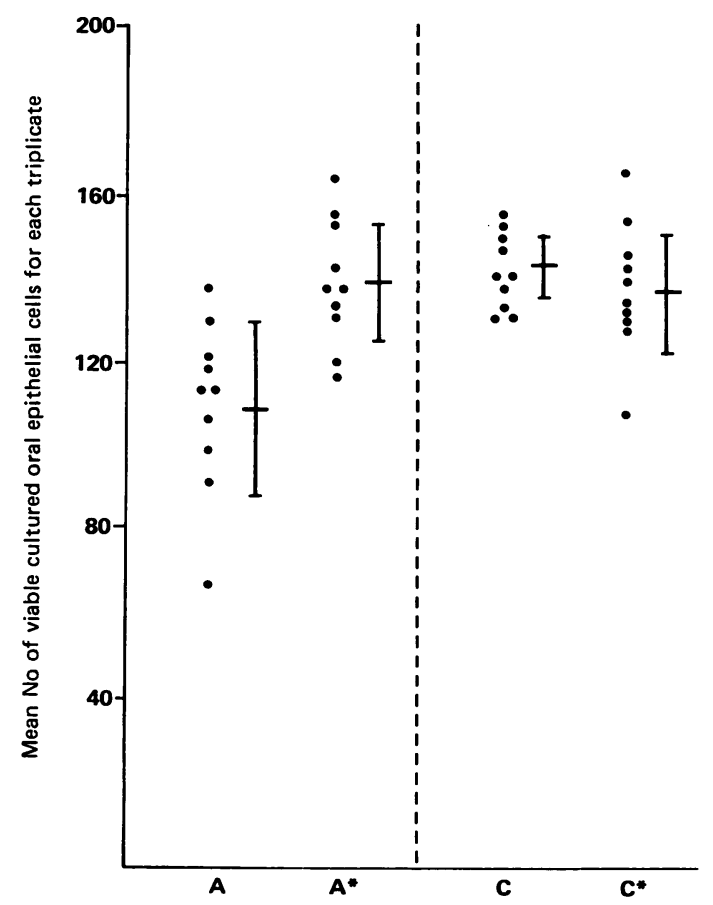

Figure 4: Mean number of cultured viable oral epithelial cells per 200 cells counted after incubation with leucocytes from aphthous patients (A) and control subjects (C) pre-and post $\left(^{\star}\right)$ pan $T$ cell depletion. The horizontal bars represent the overall mean values and corresponding standard deviations.
EFFECT OF DEPLETION OF NK CELLS ON CYTOTOXICITY

The leucocytes of a further seven patients with aphthous ulceration were more cytotoxic for the epithelial targets than those from healthy controls $(\mathrm{p}<0 \cdot 01)$. Leucocyte pretreatment with anti Leu-11 and complement, to deplete the population of NK cells, did not significantly reduce this enhanced cytotoxicity ( $\mathrm{p}>0 \cdot 1)$ (Fig 5$)$.

\section{Discussion}

All of the in vitro cytotoxicity assays described in studies of recurrent aphthous ulceration have disadvantages which limit comparisons with the in vivo situation. Burnett and $\mathrm{Wray}^{8}$ have pointed out that the cytotoxic activity of peripheral blood leucocytes may not be representative of that exerted by the leucocytes in the aphthous lesion. In addition, experimental manipulation of the target cells may alter their surface antigenic structure and therefore affect leucocyte recognition which is an essential preliminary step in cytotoxicity.

By using cultured epithelial cells dispersed by a very brief trypsinisation, the present study attempted to minimise any changes in the surface of the target cells, whereas previous studies have harvested oral epithelial cells from biopsies by treatment with trypsin for periods of time ranging from two hours ${ }^{5}$ to 12 hours. ${ }^{14}$ Furthermore, in this study the trypsinised epithelial cells were subsequently allowed to recover in medium for two hours and had a viability of at least $80 \%$, as against that in some previous investigations where the corresponding value was as low as $55 \% .^{14}$

Although the surface antigens specific to

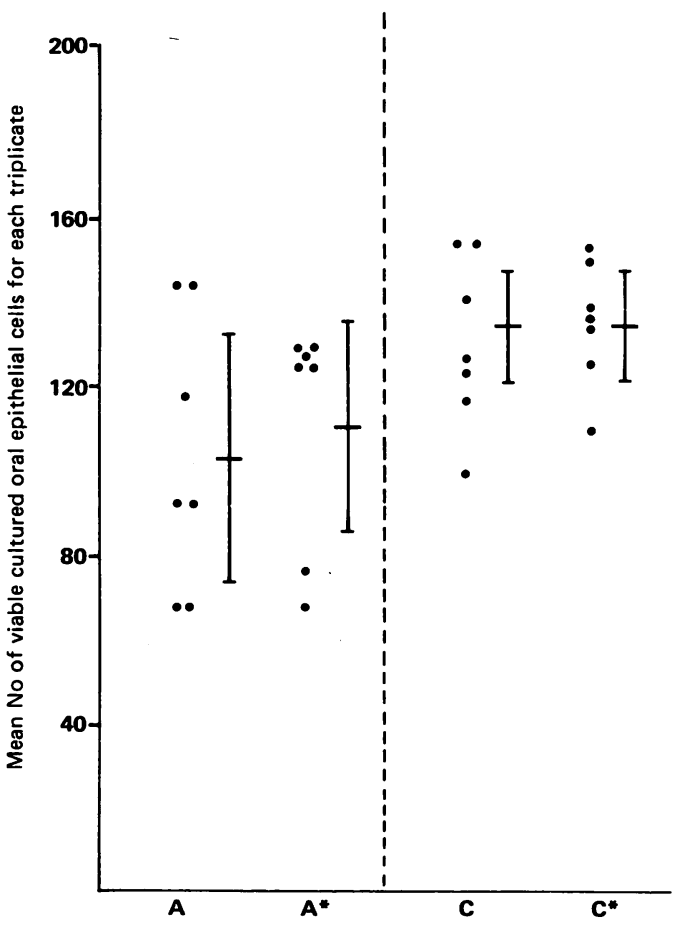

Figure 5: Mean number of cultured viable oral epithelial cells per 200 cells counted after incubation with leucocytes from aphthous patients (A) and control subjects (C) pre-and post $\left(^{\star}\right)$ NK cell depletion. The horizontal bars represent the overall mean values and corresponding standard deviations. 
squamous epithelium were preserved in the cultured cells, as recognised by pemphigus serum and antikeratin antibodies, epithelial cell surfaces may be considerably altered in long term culture. ${ }^{15}$ It is also possible that selective proliferation of a particular unrepresentative epithelial clone could occur in culture.

Assays of cell mediated cytotoxicity in recurrent aphthous ulceration have included dye exclusion, ${ }^{45}{ }^{16}$ measurement of ${ }^{51} \mathrm{Cr}$ release from the target cells ${ }^{814}$ and automated cell counting systems. ${ }^{6}$ In this study, dye exclusion, using ethidium bromide and acridine orange as the staining media, was used. The dichromism in this system was preferred to 'one colour' dye exclusion techniques, such as with trypan blue,${ }^{4}$ in which interpretation may be difficult.

A disadvantage of visual assay systems is their degree of inherent subjectivity. To eliminate interexaminer variability in this study, all cytotoxicity assays were read microscopically by one examiner. Assays involving radiolabelling techniques provide a means of eliminating subjectivity, but labelling epithelial cells with radioisotopes is difficult. Excessive spontaneous release of ${ }^{51} \mathrm{Cr}$ from oral epithelial cells has been reported by other workers ${ }^{814}$ which limited the incubation periods to a maximum of eight hours. This could explain why enhanced cytotoxicity in recurrent aphthous ulceration could not be shown in those studies, ${ }^{814}$ as successful attempts to demonstrate an in vitro cell mediated cytotoxic effect in recurrent aphthous ulceration have used incubation times of between 18 and 24 hours. ${ }^{+5616}$

Statistical analysis of the results by the F test, for the initial five leucocyte/epithelial cell cocultures showed that at an effector:target cell ratio of 200:1 the survival of the target cells was significantly reduced in the presence of aphthous compared with control leucocytes $(p<0.05)$, in agreement with previous reports. ${ }^{456}$ This difference was greater than that within each triplicate, showing that the assay system was sufficiently discriminatory for the purposes of the investigation. The 200:1 effector to target cell ratio was adopted for all subsequent experiments.

The enhanced in vitro cytotoxicity of peripheral blood leucocytes from aphthous patients compared with controls was evident both during periods of active ulceration and in remission. The raised cytotoxic activity was, however, more marked when blood was taken from patients in a phase of active ulceration rather than during an ulcer free period. This correlation between cytotoxic activity and exacerbation of the disease has been noted before ${ }^{56}$ and represents evidence for a primary aetiological role for cell mediated cytotoxicity in the disease process. The cytotoxic activity detected was independent of the presence of serum factors, in agreement with the findings of Rogers et al, ${ }^{56}$ but in contrast with that observed by Burnett and Wray. ${ }^{8}$ Greenspan et $a l^{7}$ have also studied antibody dependent cellular cytotoxicity in recurrent aphthous ulceration, and found that although there was an increase in antibody dependent cellular cytotoxic activity at the early stage of the disease, there was no increase over control levels at any other stage of the ulcerative process. We, there- fore, believe that the serum independent cytotoxicity reported in this paper is likely to be at least as significant as antibody dependent cellular cytotoxic activity in the pathogenesis of aphthous ulceration.

The failure to detect significant differences in the leucocyte c jtotoxicity of patients with types of mouth ulceration other than aphthae is not in keeping with the proposal of Burnett and Wray ${ }^{8}$ that the enhanced in vitro cell mediated cytotoxicity in recurrent aphthous ulceration towards oral epithelial cells is an epiphenomenon secondary to chronic epithelial damage. The fact that recurrent aphthous ulceration patients in remission showed leucocyte cytotoxicity greater than that for patients with active non-specific ulceration lends further support to the theory that enhanced leucocyte cytotoxicity may play a primary role essential to the pathogenesis of aphthae.

Dolby ${ }^{16}$ showed that the enhanced spontaneous cell mediated cytotoxic effect of peripheral blood leucocytes from recurrent aphthous ulceration patients for oral epithelial cells could be blocked by preincubating the peripheral blood leucocytes with polyclonal antilymphocyte serum. To investigate this finding in more detail in the present study, the leucocyte preparations were selectively depleted of leucocytes bearing the CD-5 antigen ( $T$ lymphocytes) and CD-16 antigen (NK and K cells). Pan T cell depletion abrogated the enhanced cytotoxicity exerted by aphthous leucocytes on oral epithelial cells, suggesting that the enhanced 'disease specific' in vitro cytotoxicity seen in patients with minor aphthous ulceration involves mediation of $\mathrm{T}$ lymphocytes.

Borst $e t a l^{17}$ recently showed that at effector to target cell ratios of greater than $30: 1$, the relative specificity of cell mediated cytotoxicity is greatly reduced. An increased level of non-specific, natural-killer (NK)-like activity of leucocytes is noted at higher effector to target cell ratios. Nevertheless, the present experiments detected a significant difference in activity between aphthous and control peripheral blood leucocytes towards epithelial target cells which correlated with disease activity.

The observed in vitro cytotoxicity in these experiments is clearly not MHC-restricted, in contrast to the classical MHC dependence of T cell mediated cytotoxicity. This lack of restriction has previously been noted by Rogers et al, ${ }^{56}$ who were able to show an in vitro cytotoxic effect of leucocytes from aphthous patients towards both syngeneic and allogeneic target cells. Current knowledge of the cellular immune system, however, has shown that cytotoxic T cells are capable of specific target cell lysis mediated independently of the MHC system, ${ }^{18}$ in keeping with our results.

Depletion of cells bearing the CD16 antigen (NK and $\mathrm{K}$ cells) produced no statistically significant decrease in the cytotoxic activity of leucocytes from patients with minor aphthous ulceration. This result implies that natural killer and killer cells are unlikely to be responsible for the enhanced cell mediated cytotoxicity in minor aphthous ulceration, in contrast with the work of Greenspan $e t a l^{7}$ which implicated such cell 
populations as possible effectors of the cell damage in recurrent aphthous ulceration.

In conclusion, this study has confirmed the enhanced cell mediated cytotoxic activity of peripheral blood leucocytes from patients with recurrent aphthous ulceration for oral epithelial cells. This activity apparently involves $\mathrm{T}$ lymphocytes rather than natural killer or killer cells, and the cytokines involved in the resulting tissue damage are now being investigated.

We are grateful to Mrs K J Davies for expert technical assistance, and to Dr T J Peters of the Department of Medical Statistics, University of Wales College of Medicine, for advice on study design and statistical analysis of the data.

1 Wray D. Aphthous ulceration. $\mathcal{F}$ S Soc Med 1984; 77 : 1-4.

Lehner T. Recurrent aphthous ulceraton and autoimmunity. Lancet 1964; ii: $1154-5$.

3 Currie $S$. Destruction of muscle cultures by lymphocytes from cases of polymyositis. Acta Neuropathol (Berlin) 1970; 15: 119 .

4 Dolby AE. Recurrent aphthous ulceration. Effect of sera and peripheral blood lymphocytes upon oral epithelial tissue culture cells. Immunology 1969; 17: 709-14.

5 Rogers RS, Sams WM, Shorter RG. Lymphocytotoxicity in recurrent aphthous stomatitis. Arch Dermatol 1974; 109: 361-3.

6 Rogers RS, Movius DL, Pierre RV. Lymphocyte-epithelial cell interactions in oral mucosal inflammatory diseases. F Invest Dermatol 1976; 67: 599-602.

7 Greenspan JS, Gadol N, Olson JA, Talal N. Antibodydependent cellular cytotoxicity in recurrent aphthous ulceration. Clin Exp Immunol 1981; 44: 603-10.

8 Burnett PR, Wray D. Lytic effects of serum and mononuclear lymphocytes on oral epithelial cells in recurrent aphthous stomatitis. Clin Immunol Immunopathol 1985; 34: 197-204.

9 Savage NW, Seymour GJ, Kruger BJ. T lymphocyte subset changes in recurrent aphthous stomatitis. Oral Surg Oral Med Oral Pathol 1985; 60: 175-81.

10 Lehner T. Autoimmunity in oral disease with special reference to recurrent oral ulceration. Proc $R$ Soc Med 1968; 61: 51524.

11 Boyum A. Isolation of mononuclear cells and granulocytes from human blood. Scand $\mathcal{F}$ Clin Lab Invest 1968; 21 (Suppl 97): 77-89.

12 Rheinwald JG, Green H. Serial cultivation of strains of human epidermal keratinocytes: the formation of keratinizing colonies from single cells. Cell 1975; 6: 331-44.

13 Culling CFA, Allison RT, Barr WT. Cellular pathology techniques. 4th ed. London: Butterworths, 1982: 369.

14 Reimer G, Steinkohl S, Djawari D, Hornstein OP. Lytic effect of cytotoxic lymphocytes on oral epithelial cells in Behçet's of cytotoxic lymphocytes on oral epithelial

15 Morhenn VB, Benike CJ, Cox AJ, Charron DJ, Engleman EG. Cultured human epidermal cells do not synthesise HLADR. F Invest Dermatol 1982; 78: 32-7.

16 Dolby AE. Mikulicz recurrent oral aphthae: the effect of anti-lymphocytes serum upon the in-vitro cytotoxicity of lymphocytes from patients for oral epithelial cells. Clin Exp Immunol 1970; 7: 681-6.

17 Borst J, Van de Griend RJ, Van Oostveen JW, et al. A T-cel receptor gamma/CD3 complex found on cloned functional lymphocytes. Nature 1987; 325: 683-8.

18 Staerz UD, Karasuyama H, Garner AG. Cytotoxic T-lymphocytes against a soluble protein. Nature $1987 ; 329$ : 449-51. 